J. Lake Sci.(湖泊科学), 2008, 20(6): 723-732

http://www.jlakes.org. E-mail: jlakes@niglas.ac.cn

(C)2008 by Journal of Lake Sciences

\title{
一个概念性水文模型的参数区域化研究：以东江流域为例"
}

\author{
靳晓莉 ${ }^{1,2}$, 张 奇 $^{1}$, 许崇育 ${ }^{1,3}$ \\ (1: 中国科学院南京地理与湖泊研究所湖泊与环境国家重点实验室, 南京 210008) \\ (2: 中国科学院研究生院, 北京 100049) \\ (3: 奥斯陆大学地球科学系, 奥斯陆 0316 , 挪威)
}

摘 要: 由于大尺度水文模型和无资料区水文研究是当前国际水文研究的重点和难点, 通过参数区域化方法来估计大尺度区 域和无资料区的模型参数值成为了研究的热点之一. 将 HBV 模型应用于东江流域及其子流域, 采用代理流域法和全局平均 法来估计该区域内无资料流域的模型参数. 研究表明: HBV 模型能较好得用于东江流域径流模拟; 交叉检验中, 较小的 $R^{2}$ 和 $M E$ 值对应的参数, 其转移效果不一定比较大的 $R^{2}$ 和 $M E$ 值对应的参数转移效果差; 全局平均法中, 面积权重平均值和泰森多 边形插值后平均并不能明显改进子流域算术平均值估计无资料流域的模型参数的模拟结果; 两者都能有效用于东江流域无资 料流域的参数估计, 且效果相差不大.

关键词: 参数区域化; 无资料区; 概念性水文模型; 东江流域

\section{Regionalization study of a conceptual hydrological model in the Donjiang Basin}

\author{
JIN Xiaoli ${ }^{1,2}$, ZHANG Qi ${ }^{1} \&$ XU Chongyu ${ }^{1,3}$ \\ (1: State Key Laboratory of Lake Science and Environment, Nanjing Institute of Geography and Limnology, Chinese Academy of \\ Sciences, Nanjing 210008, P.R.China) \\ (2: Graduate School of Chinese Academy of Sciences, Beijing 100049, P.R. China) \\ (3: Department of Geosciences, University of Oslo, P.O. Box 1047 Blindern, 0316 Oslo, Norway)
}

Abstract: The conceptual rainfall-runoff model, HBV, was applied to Dongjiang basin and its 13 sub-basins for the purposes of examining the applicability of this well-known model in south China in order to explore the possibility of transferring the calibrated parameter values to ungauged basins. For testing the applicability of the model in gauged basins, an automatic optimization method was used for model calibration. For testing the transferability of the parameter values to ungauged basins, two parameter regionalization methods (proxy basin and global mean) to determine the model parameters for ungauged catchments in the study area were investigated. The results showed that: (1) the HBV model worked well in the Dongjiang basin with average $R^{2}$ higher than 0.82 for using calibrated values; (2) Transferring the parameter values with higher $R^{2}$ and $M E$ during cross-basins tests into the hypothetic ungaged catchments did not result in better model simulations than those with lower corresponding values; (3) Neither area-weighted-mean nor Thiessen-interpolated-mean approach could markedly improve the precision of parameters estimation by arithmetic mean value. It was concluded that both regionalization methods could effectively estimate parameters for ungauged catchments in the Dongjiang basin, leading to little discrepancy in the two models.

Keywords: Parameter regionalization; ungaged catchments; conceptual hydrological model; Dongjiang Basin

在过去的几十年里, 随着陆地尺度的水文演变, 气候变化的水文响应研究和大尺度水资源评估使得 一种尺度介于大气循环模型 $(\mathrm{GCMs})$ 和流域水文模型之间的大尺度水文模型(MHMs)越来越重要. Arnell ${ }^{[1]}$ 总结了大河流域上模拟重要的原因: 1)为了实施和规划的目的, 水资源管理者需要估计管理区域内水资源

* 中国科学院 “百人计划” 项目和香港研究资助局项目(CUHK4627/05H)联合资助. 2007-12-17 收稿; 2008-04-10 收修改 稿. 靳晓莉, 女, 1980 年生, 博士研究生; E-mail: yezi1612@hotmail.com. 
的空间变化, 其空间分辨率要求比观测数据更高; 2)水资源管理者和水文学家都很关注大的地理范围内 土地利用和气候变化对水文过程所产生的影响; 3)水文模型能有效估计入河点源和非点源污染; 4)水文学 家和大气模拟者都已清晰地认识到区域和全球大气模型中对于水文过程描述的弱点, 并希望做出改进. 但是, 这种以大尺度区域作为研究对象的模型模拟，由于大尺度上的数据信息获取的困难，一般很难通 过实测数据来率定模型参数 ${ }^{[2]}$, 只能通过小尺度上参数值或者区域的物理属性信息获得. 另一方面, 无 资料流域的水文变量的预测是水文科学的主要热点问题之一 ${ }^{[3]}$. 当把现有的水文模型应用于无资料区(PUB) 的研究时，由于观测资料的不完整或完全缺失，也使得模型的参数值很难通过率定的方法来确定，而只能 通过有资料区的参数值或者无资料区的自然属性信息来估计. 将有资料区的信息向无资料区转移 ${ }^{[4-5]}$, 或者 由小尺度的模型参数获取较大尺度的模型参数 ${ }^{[2]}$ 正是参数区域化所要研究的问题. 参数区域化的目的是 对任一栅格、子流域或者大区域上的水文模型, 无需通过率定或者手动调整而能够获得模型参数值 ${ }^{[2]}$.

当前, 国外学者已经进行了大量的参数区域化研究, 其中主要采用了以下几类方法: 1)“代理”流域法 (Proxy basins) $)^{[6-7]}$; 2) 空间插值法: 线性插值 ${ }^{[8]}$, 克里格插值 ${ }^{[9]} ; 3$ ) 聚类分析法 ${ }^{[10-11]} ; 4$ 二元或多元回归方法 $\left.{ }^{[7,12]} ; 5\right)$ 一步回归方程区域率定 ${ }^{[13]}$, 即同时率定水文模型和回归模型. 其中, 以多元回归方法最为常见. 该方法主要在优化的模型参数值和流域自然属性值(如流域面积, 平均坡度, 降雨特征值, 土地利用类型, 土壤类型等)之间建立多元回归方程，无资料区的参数值由自身的自然属性和回归方程得到. 但是，这一 方法的应用需要较多的数据支持. 为了进行有意义的统计学回归分析, 用于优化模型参数和建立回归方 程的有资料流域必须不少于 20 个, 最少不能少于 10 个 $^{[2]}$. 另外, 它还存在如下局限性: 1)模型参数很难确 定，有些相互之间存在很强的关联性，因此这些参数本身很不确定 $\left.{ }^{[14]} ; 2\right)$ 一些模型参数很难通过区域化的 关系式来确定，换句话说，它们与可测量的物理值之间没有很强的联系 ${ }^{[15]}$. 虽然Hundecha等 ${ }^{[16]}$ 和 Gotzinger等 ${ }^{[17]}$ 通过首先假定回归方程的形式, 然后通过实际观测数据不率定水文模型参数而直接率定回 归方程的参数得到回归方程, 从而来避免模型参数的不确定性问题, 但是对于回归方程的形式的假定并 不能得到证明, 并且上述第二点的局限性依然存在. 例如Braun和Renner ${ }^{[18]}$ 研究了 HBV 模型的参数和流 域特征之间的关系，再将这个模型应用于瑞士不同部分的 5 个流域中，总结得出流域特征和模型参数之 间没有联系的结论. Johansson ${ }^{[19]}$ 研究了模型参数值和瑞典南部的 11 个流域的 12 个流域特征之间的关系后 发现, 只有一个参数和流域特征之间存在联系. 在参数区域化研究中, 随着单个研究方法的深人, 也有 学者将多个方法同时应用, 进行了比较. Vandewiele和Elias ${ }^{[9]}{ }^{1}$ 发现克里格方法能改进多元回归方法估计月 水量平衡模型在无资料区的参数. Kokkonen等 ${ }^{[20]}$ 认为当有理由相信一个有资料流域与一个无资料流域在 水文行为方面相似时，那么采用有资料流域全部的率定的参数，而不是去获取模型参数和流域特征之间 定量关系的做法是值得的. 值得一提的是, Merz和Blosch ${ }^{[21]}$ 在澳大利亚 308 个流域中对于一个概念性流 域模型检查了 8 种参数区域化方法的效果，得出结论：基于空间邻近性的区域化方法(如克里格插值法)比 任何一种基于流域属性的方法(如全局回归和局部回归)效果更好. Parajka ${ }^{[22]}$ 对4组共7种区域化方法的研 究得出了与上述几个研究相同的结论.

选择珠江流域的一个子流域一一东江流域为研究区, 将 HBV 模型应用于东江流域及其子流域, 并且 采用了两类区域化方法来估计该研究区内无资料流域模型的参数值, 目的在于：1)检查 HBV 模型在中国 南部流域的适用性; 2)寻求一种尽可能简单的、数据需求少而应用效果好的参数区域化方法用于估计东江 流域内无资料区的模型参数. 国内对于参数区域化的研究相对于国外要少很多, 本文有意在这方面做些 尝试和探索, 研究结果将对东江流域乃至整个珠江流域的无资料区或者大尺度流域的模型参数估计提供 借鉴和参考. 同时, 东江流域作为珠三角(包括香港)的供水源地之一，提供了香港总供水量的 $80 \%$ 的水， 流域水资源量的多少和变化将对整个珠三角社会经济发展起到举足轻重的作用. 模型的模拟和区域化方 法的研究将可能对东江流域水资源评估和管理提供决策支持.

\section{1 研究区}

东江是中国南部珠江的一个支流，发源于江西省寻邬县，自东北向西南注人珠江口，干流总长 $562 \mathrm{~km}$. 东江流域位于广东省和江西省内, 博罗站以上流域面积为 $25555 \mathrm{~km}^{2}$ (后文中不做特殊说明的, 东 
江流域面积均为博罗站控制面积). 流域平均坡度 $0.39 \%$, 地形以丘陵和平原为主, 分别占整个流域面积 的 $78.1 \%$ 和 $14.4 \%$. 高海拔地区为森林覆盖, 而低海拔的丘陵和平原地区则主要是农业用地. 流域气候为 亚热带气候，年平均温度为 $21^{\circ} \mathrm{C}$, 只有流域上游一些山区冬季的日温度偶尔会低于 $0^{\circ} \mathrm{C}$. 多年平均降雨量 (1960-1988 年)为 $1747 \mathrm{~mm}$, 年平均流量 $935 \mathrm{~mm}$, 年径流系数为 0.54 . 降雨类型主要为锋面雨和台风降雨 两类. 降雨和径流的季节变化很大, $80 \%$ 的年降雨和径流发生在四月至九月的湿季, 剩下的 $20 \%$ 集中在 10 月至次年 3 月的干季.

研究不仅包括了整个东江流域，也包括了其中有流量观测数据的 13 个自然子流域. 这些子流域出口 断面控制面积从几十平方千米到上千平方千米不等, 有些是独立的, 有些具有嵌套关系，在流域上、中、下 游都有分布. 东江流域和子流域如图 1 所示. 所采用的数据包括流域内 51 个雨量站的日降雨量、8 个站的 日平均温度、5 个站的日潜在蒸发量、14 个流量站的日流量, 数据自 1978-1988 年共 11 年. 东坑和红花塔 子流域由于流量数据的缺乏, 只截取了 1978-1980 年共 3 年的数据, 在研究和分析中不作为重点.

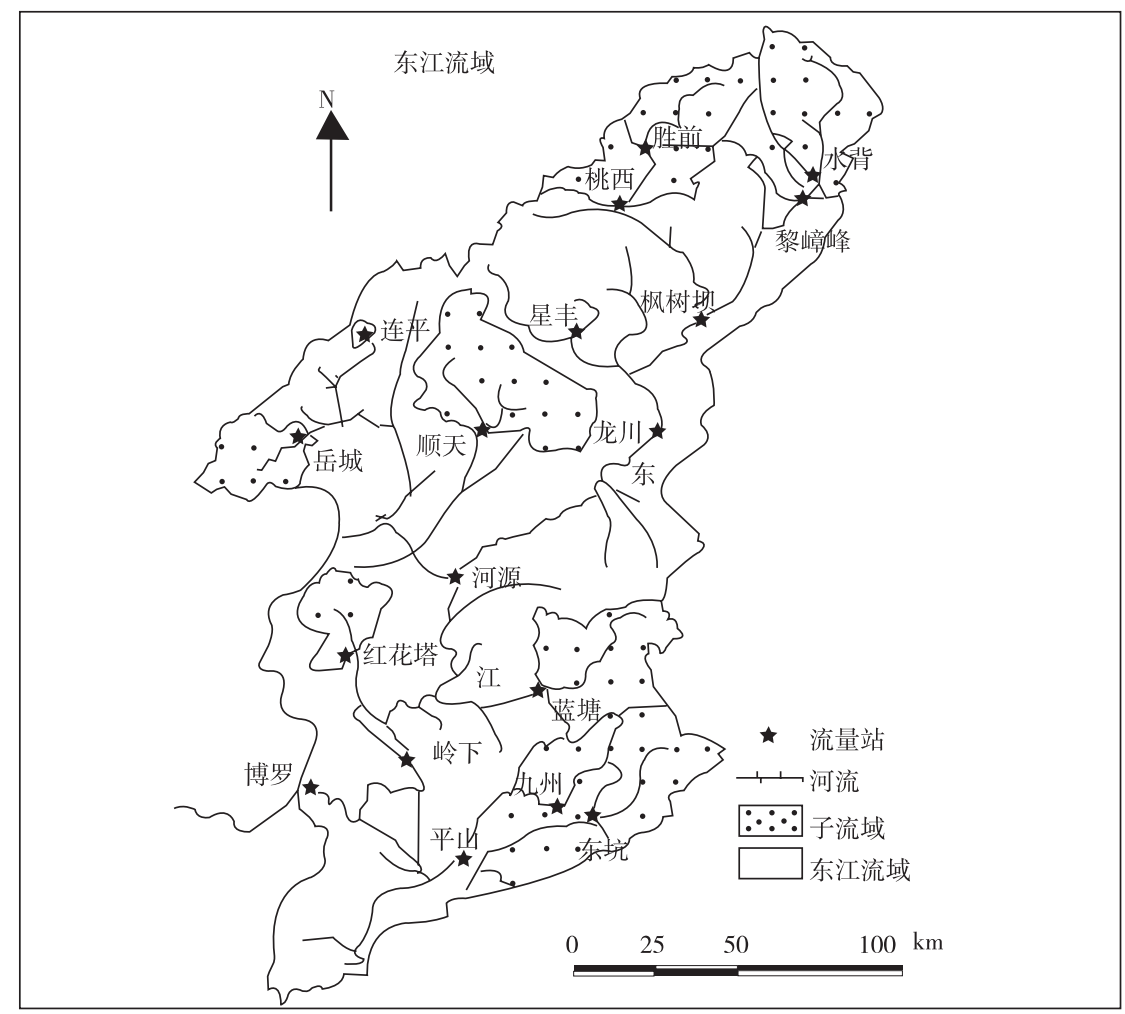

图 1 东江流域及其子流域图

Fig.1 The map of Dongjiang Basin and sub-basins

\section{HBV 模型}

$\mathrm{HBV}$ 模型 ${ }^{[23]}$ 是由SMHI(瑞典气象和水文研究所)开发. 在过去30多年里, 随着推广和应用, 不断推出 新的版本. 本研究所采用的模型版本一 - HBV light ${ }^{[24]}$ 在Bergstrom ${ }^{[25]}$ 所描述的HBV-6版本的基础上, 进 行了两处改进.

HBV模型是一个概念性流域水文模型. 输人数据为日降雨量、气温和月潜在蒸发量, 输出为日流量. 模型包括融雪模块(用日温度方法表示积雪和融雪)，土壤模块(其中地下水补给和实际蒸发用实际土壤蓄 水量的函数来计算), 响应模块(用三个线性水库方程描述)和一个路径模块(三角权重函数表示). 模型共 有 13 个参数. 主要的计算公式如下: 
融雪模块:

$$
\begin{aligned}
& \text { melt }=C F M A X(T(t)-T T) \\
& \text { refreezing }=C F R \quad C F M A X(T T-T(t))
\end{aligned}
$$

土壤模块:

$$
\begin{aligned}
& E_{\mathrm{act}}=E_{\mathrm{pot}} \min \left(\frac{S M(t)}{F C \cdot L P}, 1\right) \\
& \frac{\text { recharge }}{P(t)}=\left(\frac{S M(t)}{F C}\right)^{B E T A}
\end{aligned}
$$

响应模块:

$$
Q_{G W}(t)=K_{2} S L Z+K_{1} S U Z+K_{0} \max (S U Z-U Z L, 0)
$$

路径模块:

$$
Q_{\text {sim }}(t)=\sum_{i=1}^{M A X B A S} c(i) Q_{G W}(t-i+1)
$$

式中: $C F R, C F M A X$ 为积雪和融雪参数; $T(t)$ 为日平均温度; $T T$ 为温度阈值参数; $E_{\mathrm{act}}, E_{\mathrm{pot}}$ 为日实际和潜在 蒸发量; $S M(t)$ 为第 $t$ 天的土壤含水量; $F C$ 为田间持水量; $L P$ 为土壤含水量与田间持水量之比的阈值; $P(t)$ 为日降雨量; BETA 为指数参数; $S U Z, S L Z$ 为上层和下层土壤含水量; $U Z L$ 为上层土壤含水量阈值; $K_{0} 、 K_{1}$ 和 $K_{2}$ 为三个出口的退水系数; $M A X B A S$ 为路径参数.

土壤模块中, 蒸散发的计算由参数 $L P$ 控制, 当土壤含水量与田间持水量的比值超过 $L P$ 时, 实际蒸 发量等于潜在蒸发量, 当比值小于 $L P$ 时, 实际蒸发量随着土壤含水量的减少呈线性递减. 进人土壤的降 雨量由参数 BETA 决定, 由于下渗过程依赖于土壤类型和土地利用类型, 因此这个参数也与土壤类型和 土地利用类型有关. 响应模块用三个线性水库表示, 相应的也由三个退水系数确定, 其中 $K_{0}$ 代表洪峰(或 高水位时的退水系数, $K_{1}$ 为壤中流的退水系数, $K_{2}$ 为基流的退水系数). 路径模块中, 最后的流量过程 还依赖于形状参数 $M A X B A S$, 这个参数与流域面积有关, 面积越大, 则 $M A X B A S$ 也会越大.

HBV 模型更详细介绍请参考 Bergstrom ${ }^{[25]}$, Hundecha and Bardossy ${ }^{[16]}$.

\section{3 参数区域化方法和结果分析}

\section{1 模型的应用}

由于结构简单, 输入数据要求少, 参数也比较少, HBV 模型被较多地应用于参数区域化研究中(包括本研究). 它是以北欧的水文环境为背景开发的, 在欧洲, 美洲, 澳洲等地区 30 多个国家都有广泛的应用, 但在国内应用还 比较少, 尤其是在南部亚热带地区. 东江流域几乎无降雪天气, 因此本研究中, 模型的融雪模块基本上被忽略. 模型在东江流域及其 13 个子流域的应用将用于检验 HBV 模型忽略融雪过程情况下, 在该地区的适用性.

模型的参数(除融雪模块的两个参数)的率定采用模型自带的 GAP 优化方法来获得. 采用下面两个指 标来分别度量均值和极值的模拟效果——确定性系数 $\left(R^{2}\right)$ 和 Nash 效率系数 $(M E)$.

$$
\begin{aligned}
& R^{2}=\frac{\left(\sum\left(Q_{\mathrm{obs}}-\overline{Q_{\mathrm{obs}}}\right)\left(Q_{\text {sim }}-\overline{Q_{\text {sim }}}\right)\right)^{2}}{\sum\left(Q_{\mathrm{obs}}-\overline{Q_{\mathrm{obs}}}\right)^{2} \sum\left(Q_{\mathrm{sim}}-\overline{Q_{\mathrm{sim}}}\right)^{2}} \\
& M E=1-\frac{\sum\left(Q_{\mathrm{obs}}-Q_{\mathrm{sim}}\right)^{2}}{\sum\left(Q_{\mathrm{obs}}-\overline{Q_{\mathrm{obs}}}\right)^{2}}
\end{aligned}
$$

式中: $Q_{\mathrm{obs}} 、 \overline{Q_{\mathrm{obs}}}$ 为观测值及观测值平均值; $Q_{\mathrm{sim}} 、 \overline{Q_{\mathrm{sim}}}$ 为模拟值及模拟值平均值. $R^{2}$ 和 $M E$ 取值范围在 $0-1$ 之间, 越接近 1 , 则模拟效果越好.

表 1 中, 除了星丰和胜前子流域外, 其它子流域 $R^{2}$ 和 $M E$ 都在 0.75 以上, 子流域平均 $R^{2}$ 和 $M E$ 分别 
达到 0.82 和 0.81 , 整个东江流域的 $R^{2}$ 和 $M E$ 高达 0.86 .

流量过程线图中(篇幅的限制，仅以博罗站为例，如图 2 和图 3)，日和月的观测值和模拟值的对比， 无论是整个东江流域还是子流域, 过程线基本一致, 模型模拟的结果与实际吻合较好. HBV 模型忽略融 雪模块，能较好地模拟出东江流域及其子流域的日径流过程.

表 $1 \mathrm{HBV}$ 模型在东江流域及其子流域模拟效果

Tab.1 Simulation results of HBV modeling in the Dongjiang Basin and sub-basins

\begin{tabular}{cccccccccc}
\hline 编号 & 名称 & 面积 $\left(\mathrm{km}^{2}\right)$ & $R^{2}$ & $M E$ & 编号 & 名称 & 面积 $\left(\mathrm{km}^{2}\right)$ & $R^{2}$ & $M E$ \\
\hline 1 & 东坑 & 849 & 0.97 & 0.96 & 8 & 胜前 & 684 & 0.71 & 0.71 \\
2 & 红花塔 & 455 & 0.71 & 0.71 & 9 & 水背 & 987 & 0.79 & 0.78 \\
3 & 九州 & 385 & 0.87 & 0.87 & 10 & 顺天 & 1357 & 0.89 & 0.88 \\
4 & 蓝塘 & 1080 & 0.85 & 0.85 & 11 & 桃西 & 1306 & 0.78 & 0.77 \\
5 & 连平 & 37.2 & 0.86 & 0.86 & 12 & 星丰 & 42.6 & 0.72 & 0.72 \\
6 & 黎嶂峰 & 1400 & 0.81 & 0.80 & 13 & 岳城 & 531 & 0.81 & 0.81 \\
7 & 平山 & 2091 & 0.87 & 0.87 & 14 & 东江流域 & 25555 & 0.86 & 0.86 \\
\hline
\end{tabular}

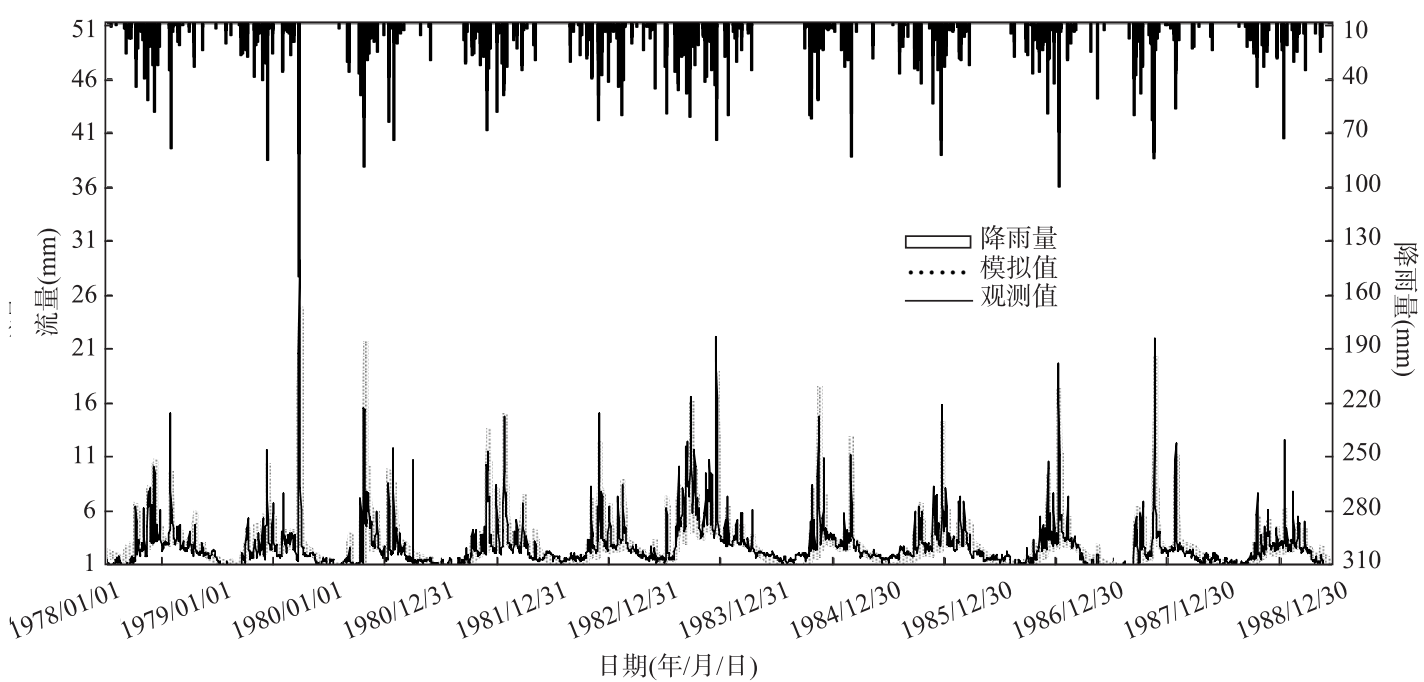

图 2 博罗站 1978-1988 年日流量和降雨量

Fig.2 Simulated and observed daily runoff and daily precipitation at Boluo station, 1978-1988

\section{2 参数区域化}

3.2.1 代理流域方法 代理流域法(Proxy basins)是先检验水文模型及其参数在水文和气候空间上具有相似 性的区域内的可转移性，然后再应用到区域内无资料流域的一种方法. 具体做法是: 如果要模拟一个无 资料流域 $\mathrm{C}$ 的径流过程，那么模型首先必须在流域 $\mathrm{A}$ 上进行率定，在流域 $\mathrm{B}$ 上进行检验，并且在 $\mathrm{B}$ 上率 定，在 A 上检验，这种交叉检验的过程也叫 Proxy-basins 检验. 只有 Proxy-basins 检验通过，才能认为模 型具有地理可转移性 ${ }^{[6]}$. 为了使通过交叉检验的子流域参数在东江流域范围内具有可转移性, 选择了流 域上游的 4 个子流域和下游的 3 个子流域进行了交叉检验，取交叉检验结果最好的两个子流域的参数来 估计其它 11 个 “无资料子流域” 的参数.

从式(7)和(8)可以看出, $R^{2}$ 能较好地反映出平均值模拟的好坏, 而 $M E$ 对于极值模拟的优劣更敏感, 为了反映出两者的综合效果, 构造变量 $F$. 则 $F$ 取值范围在 0-1 之间, 越大表示两者模拟的综合效果越好.

$$
F=R^{2} \times M E
$$




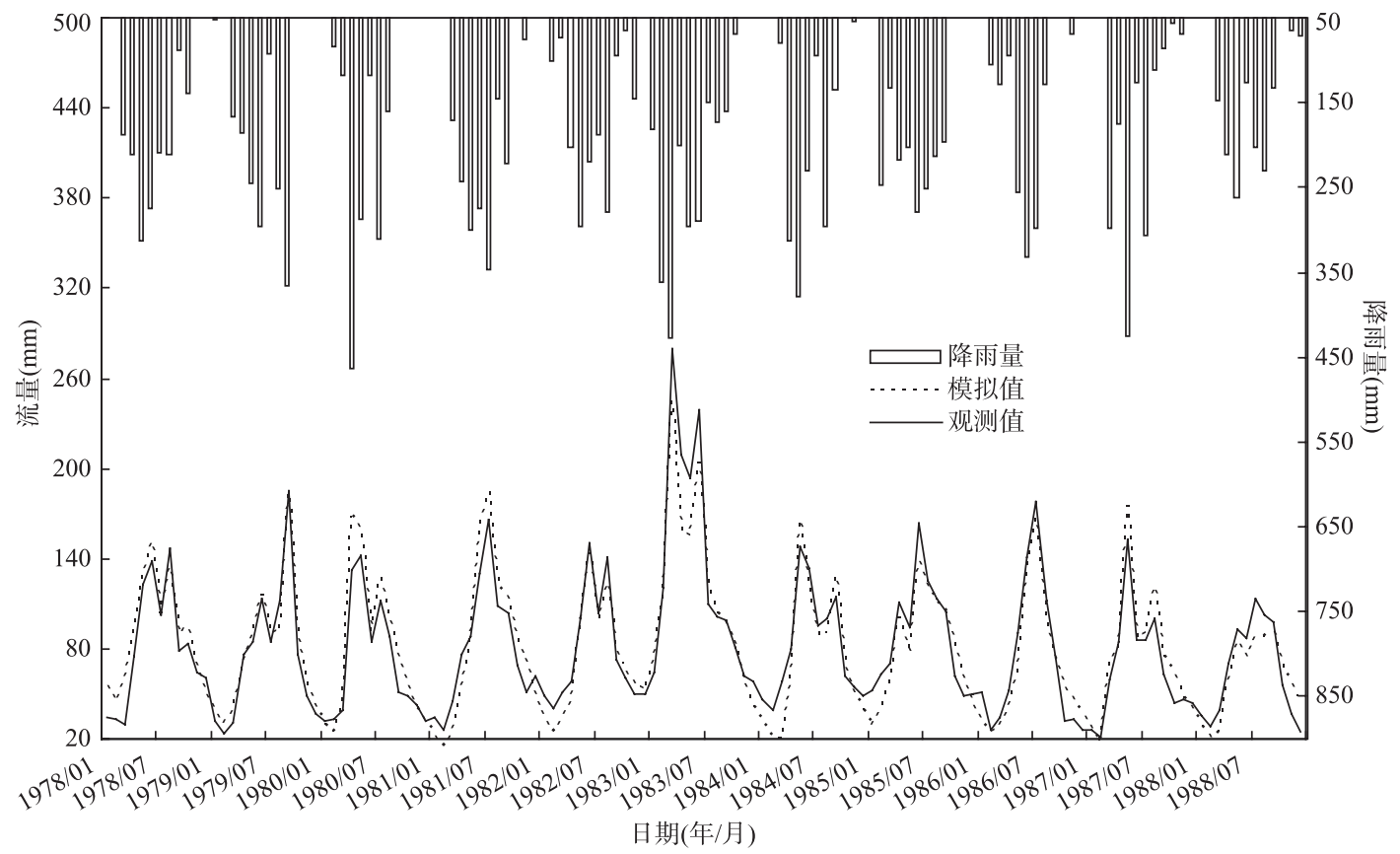

图 3 博罗站 1978-1988 年月流量和降雨量

Fig.3 Simulated and observed monthly runoff and monthly precipitation at Boluo station

交叉检验的结果如表 2 和表 3 , 九州与黎嶂峰和九州与水背两组子流域交叉检验结果要明显好于其它 几组, 两组之间相差不大. 但将黎嶂峰和水背子流域参数分别应用于其它两个下游子流域进行检验时(表 3), 水背子流域参数对应的 $F$ 值要稍高于黎嶂峰子流域参数, 因此选择将九州和水背子流域的参数转移, 来 估计其它 11 个子流域的参数. 参数转移的结果如表 4 所示.

表 2 上游子流域和下游子流域交叉检验一(下游率定，上游检验)

Tab.2 Cross-basins tests between upstream and downstream sub-basins (calibration in downstream and validation in upstream)

\begin{tabular}{|c|c|c|c|c|c|c|c|c|c|}
\hline \multirow{2}{*}{ 上游 } & \multicolumn{3}{|c|}{ 九州 } & \multicolumn{3}{|c|}{ 蓝塘 } & \multicolumn{3}{|c|}{ 平山 } \\
\hline & $R^{2}$ & $M E$ & $F$ & $R^{2}$ & $M E$ & $F$ & $R^{2}$ & $M E$ & $F$ \\
\hline 黎嶂峰 & 0.76 & 0.76 & 0.57 & 0.78 & 0.75 & 0.59 & 0.72 & 0.72 & 0.52 \\
\hline 胜前 & 0.64 & 0.60 & 0.38 & 0.67 & 0.67 & 0.45 & 0.61 & 0.57 & 0.35 \\
\hline 水背 & 0.74 & 0.73 & 0.54 & 0.75 & 0.68 & 0.51 & 0.70 & 0.70 & 0.49 \\
\hline 桃西 & 0.71 & 0.70 & 0.50 & 0.72 & 0.65 & 0.47 & 0.70 & 0.70 & 0.49 \\
\hline
\end{tabular}

表 3 上游子流域和下游子流域交叉检验二(上游率定, 下游检验)

Tab.3 Cross-basins tests between upstream and downstream sub-basins (calibration in upstream and validation in downstream)

\begin{tabular}{|c|c|c|c|c|c|c|c|c|c|}
\hline \multirow{2}{*}{ 上游 } & \multicolumn{3}{|c|}{ 九州 } & \multicolumn{3}{|c|}{ 蓝塘 } & \multicolumn{3}{|c|}{ 平山 } \\
\hline & $R^{2}$ & $M E$ & $F$ & $R^{2}$ & $M E$ & $F$ & $R^{2}$ & $M E$ & $F$ \\
\hline 黎嶂峰 & 0.84 & 0.81 & 0.68 & 0.82 & 0.81 & 0.67 & 0.68 & 0.54 & 0.37 \\
\hline 胜前 & 0.82 & 0.78 & 0.64 & 0.84 & 0.83 & 0.69 & 0.77 & 0.54 & 0.41 \\
\hline 水背 & 0.84 & 0.82 & 0.69 & 0.81 & 0.80 & 0.65 & 0.71 & 0.61 & 0.43 \\
\hline 桃西 & 0.78 & 0.78 & 0.61 & 0.82 & 0.80 & 0.66 & 0.83 & 0.78 & 0.64 \\
\hline
\end{tabular}


表 4 九州和水背子流域参数转移结果

Tab.4 Transfer results of calibrated parameters in the Jiuzhou and Shuibei sub-basins

\begin{tabular}{|c|c|c|c|c|c|c|c|c|c|c|c|}
\hline \multirow{2}{*}{$\begin{array}{l}\text { 编 } \\
\text { 号 }\end{array}$} & \multirow{2}{*}{ 名称 } & \multicolumn{2}{|c|}{ 率定 } & \multicolumn{4}{|c|}{ 九州子流域参数 } & \multicolumn{4}{|c|}{ 水背子流域参数 } \\
\hline & & $R^{2}$ & $M E$ & $R^{2}$ & $M E$ & $\mathrm{EL}\left(R^{2}\right)$ & $\mathrm{EL}(M E)$ & $R^{2}$ & $M E$ & $\mathrm{EL}\left(R^{2}\right)$ & $\operatorname{EL}(M E)$ \\
\hline 1 & 东坑 & 0.97 & 0.96 & 0.91 & 0.89 & 0.05 & 0.07 & 0.91 & 0.91 & 0.05 & 0.06 \\
\hline 2 & 红花塔 & 0.71 & 0.71 & 0.66 & 0.63 & 0.05 & 0.08 & 0.71 & 0.68 & 0.00 & 0.03 \\
\hline 3 & 九州 & 0.87 & 0.87 & 0.87 & 0.87 & 0.00 & 0.00 & 0.84 & 0.82 & 0.03 & 0.05 \\
\hline 4 & 蓝塘 & 0.85 & 0.85 & 0.81 & 0.79 & 0.04 & 0.07 & 0.81 & 0.80 & 0.04 & 0.05 \\
\hline 5 & 连平 & 0.86 & 0.86 & 0.81 & 0.78 & 0.04 & 0.07 & 0.82 & 0.79 & 0.04 & 0.06 \\
\hline 6 & 黎嶂峰 & 0.81 & 0.80 & 0.76 & 0.76 & 0.05 & 0.05 & 0.80 & 0.79 & 0.01 & 0.01 \\
\hline 7 & 平山 & 0.87 & 0.87 & 0.71 & 0.63 & 0.16 & 0.24 & 0.71 & 0.61 & 0.17 & 0.26 \\
\hline 8 & 胜前 & 0.71 & 0.71 & 0.64 & 0.60 & 0.07 & 0.11 & 0.67 & 0.64 & 0.05 & 0.07 \\
\hline 9 & 水背 & 0.79 & 0.78 & 0.74 & 0.73 & 0.05 & 0.05 & 0.79 & 0.78 & 0.00 & 0.00 \\
\hline 10 & 顺天 & 0.89 & 0.88 & 0.79 & 0.69 & 0.09 & 0.19 & 0.84 & 0.75 & 0.05 & 0.13 \\
\hline 11 & 桃西 & 0.78 & 0.77 & 0.71 & 0.70 & 0.07 & 0.07 & 0.75 & 0.74 & 0.03 & 0.03 \\
\hline 12 & 星丰 & 0.72 & 0.72 & 0.69 & 0.64 & 0.03 & 0.08 & 0.65 & 0.55 & 0.08 & 0.17 \\
\hline 13 & 岳城 & 0.81 & 0.81 & 0.81 & 0.77 & 0.01 & 0.04 & 0.78 & 0.72 & 0.03 & 0.09 \\
\hline
\end{tabular}

用九州子流域参数估计其它子流域参数, 各子流域实测数据来检验, $R^{2}$ 最小值为 $0.64, M E$ 的最小值 为 0.60 ; 同样, 将水背子流域的参数进行转移时, $R^{2}$ 和 $M E$ 最小值分别为 0.65 和 0.55 . 在用这两组参数估 计东江流域内其它无资料区的参数时, $R^{2}$ 不会低于 $0.64, M E$ 不会低于 0.55 , 可以达到应用的要求.

仅从最小值无法准确地判断出这组参数的转移效果, 因为观测数据的错误或者模型结构的不合理 等等其它一些原因也会造成模型模拟的 $R^{2}$ 和 $M E$ 值偏低. 为了消除这些因素的影响, 我们采用“效率损 失”来分析. “效率损失”(Efficiency Loss，EL)定义为模型在该流域所能达到的最高的效率指标，即率定时 的 $R^{2}$ 和 $M E$ ，与区域化方法估计的模型参数对应的模拟效率指标之差，包括 $R^{2}$ 损失和 $M E$ 损失. 将九州 子流域参数进行转移, “无资料”子流域 $R^{2}$ 和 $M E$ 损失最大值分别为 0.16 和 0.24 , 水背子流域参数对应的 $R^{2}$ 和 $M E$ 损失最大值分别为 0.17 和 0.26 , 两者相差不大, 并且都出现在率定效果较好的平山子流域(表 4). 但在率定效果很差的胜前、星丰子流域上，两组参数对应的效率损失却有一定的差别，尤其是在星丰子 流域上, 水背子流域参数对应的效率指标损失是九州子流域参数的两倍还多. 在实际应用中, 即将这两 组参数用于估计东江流域中无资料流域的参数时，假如该流域在有实测数据情况下，率定效果很好，即 使 $R^{2}$ 和 $M E$ 损失值能高达 0.15 和 0.2 以上, 用实测数据检验参数估计对应的模拟结果, $R^{2}$ 和 $M E$ 仍能达 到 0.6 以上，模拟结果基本符合实际情况. 但是假如该无资料流域率定效果本来就差，用九州子流域参数 来估计时, 模拟结果中 $R^{2}$ 和 $M E$ 损失值为 0.03 和 0.08 , 而用水背子流域参数估计时, $R^{2}$ 和 $M E$ 损失值为 0.08 和 0.17 , 后者估计的参数模拟结果显然跟实际相差太远. 因此, 在估计东江流域中其它无资料流域 的参数时，用九州子流域率定的参数会比用水背子流域率定的参数要稍好.

值得注意的是，在九州和水背子流域参数进行交叉检验时，九州子流域参数对应的 $R^{2}$ 和 $M E$ 为 0.74 和 $0.73, F$ 值为 0.54 ; 水背对应的 $R^{2}$ 和 $M E$ 为 0.84 和 $0.82, F$ 值为 0.69 , 要明显高于前者, 但从两组参数 转移效果来看, 无论是转移后的 $R^{2}$ 和 $M E$ 最小值还是损失值, 九州子流域参数转移效果并不差于水背, 甚至略好. 因此, 交叉检验中, 较小的 $R^{2}$ 和 $M E$ 值对应的参数, 其转移效果不一定差于较大的 $R^{2}$ 和 $M E$ 值对应的参数.

当然转移效果与被转移子流域和目标子流域的地理背景条件差异存在着很大的关系，两者在流域 面积、坡度、土地利用、土壤类型等流域自然特征方面差异大，则这些特征对应的模型参数值也会存在 较大的差异，在不考虑模型参数敏感性、“异参同效”性等其它情况下，则转移效果也必然会差. 因此代理 流域法在一定程度上可以归结为参数与流域自然特征之间的关系的研究上. 目前, 在这一方面已有一些 
结论，如 Seibert ${ }^{[12]}$ 通过位于瑞典的 11 个流域的三个特征一一面积、湖泊占流域面积的比例、森林覆盖率, 和 HBV 模型的参数之间的关系的研究发现: 退水系数 $K_{1}$ 与湖泊面积所占比例、流域面积呈负相关, $K_{2}$ 随着湖泊所占比例、森林覆盖率增大而减小, 土壤模块的参数 $F C 、 B E T A$ 与湖泊所占比例存在着正相关 关系, 此外, BETA 还随着流域面积的增大而增大. 从本研究的转移结果和各子流域率定的参数值、子流 域的自然特征之间的关系来看, 基本符合上述结论. 以九州子流域和顺天子流域为例. 九州子流域面积 $385 \mathrm{~km}^{2}$ ，林地占子流域面积的 $78.9 \%$, 水体所占比例为 $0.53 \%$ (1986 年); 而顺天子流域面积为 $1357 \mathrm{~km}^{2}$, 林地和水体分别占子流域面积的 $45.63 \%$ 和 $0.59 \%$ (1986 年). 受此影响, 两个子流域率定的参数之间存在 着很大的差距, 如 $K_{1}$ 和 $K_{2}$ 在九州子流域值分别为 0.502 和 0.003 , 在顺天子流域为 0.309 和 0.053 (所有子 流域率定的 $K_{1}$ 值所在的范围为 $0.14-0.51, K_{2}$ 值所在的范围为 0.003-0.093), 从而将九州子流域率定的参 数转移到顺天子流域时, 效率损失较大, $\mathrm{ME}$ 损失为 0.19 . 但是需要再提一下的是, 转移效果的优劣不仅 取决于两个流域的自然特征差异, 还与模型参数的敏感性、不确定性等有关. 如九州子流域和岳城子流 域. 岳城子流域面积为 $531 \mathrm{~km}^{2}$, 林地和水体分别占子流域面积的 $75.32 \%$ 和 $0.53 \%$ (1986 年). 虽然两者自 然条件背景差异不大, 但率定的参数却相差很大, 如 $K_{1}$ 和 $K_{2}$ 在岳城子流域分别为 0.283 和 0.032 , 参数转 移依然能取得较好的效果, $M E$ 损失仅为 0.04 , 这说明模型参数存在着明显的“异参同效”性.

3.2.2 全局平均法 全局平均法也是一种比较简单的区域化方法，一般将大的流域内所有有资料的子流域 率定的参数算术或加权平均，直接应用于该大流域内其它的无资料子流域或者整个大流域. 在水文模型 中, 流域的属性特征是用参数来表征. 参数的算术平均值也即流域平均属性特征. 虽然 Merz 等 ${ }^{[21}$ 和 Parajka 等 ${ }^{[22]}$ 通过各自的比较研究都认为全局平均法是多种区域化方法中效果最差的一种, 但是由于本 研究选取的东江流域比上述研究中选取的研究区范围小很多, 子流域属性相差也小, 参数平均后与实际 的子流域参数相差也会很小, 因此这种方法依然值得一试.

算术平均法不考虑子流域面积，也不考虑子流域分布的位置，但作者认为，面积大的子流域包含的 流域平均信息多，应该突出其作用，因此，按照子流域的面积大小来给定参数的权重值. 面积大的子流 域参数, 权重值大, 相反面积小的子流域参数, 权重值小. 得到另一组以面积大小决定权重的流域平均 参数值, 简称为“面积权重平均值”.

同样, 考虑子流域的分布位置及稀疏程度, 进行参数的空间插值, 然后得到整个大流域的平均参数 值. 插值方法采用泰森多边形法, 表示位于大流域中间的以及分布稀疏地区中的子流域参数值, 对流域 平均参数值的影响要大于位于大流域边缘的以及分布密集地区中的子流域. 得到的一组插值后的流域平 均参数值简称为“泰森插值平均值”.

三组流域平均值分别应用于 13 个子流域和整个东江流域(表 5)表明, 三组参数平均值所对应的子流 域模拟结果中, 只有率定效果最差的星丰子流域的 $M E$ 小于 0.6 , 其它子流域的 $\mathrm{ME}$ 和所有的 $R^{2}$ 都超过了 0.6 , 该方法也能有效用于东江流域无资料区的参数估计. 三组平均值中, 无论采用哪种, $R^{2}$ 和 $\mathrm{ME}$ 最小值 都出现在率定时效果最差的星丰子流域上，在该子流域上，三组平均值对应的 $R^{2}$ 和 $M E$ 仅有细微的差别.

以效率损失来分析, 三组平均值, 对应的 $M E$ 损失都要大于 $R^{2}$ 损失. $R^{2}$ 损失最大值为 $0.12, M E$ 损失 最大的能达到 0.26. 三组平均值对应的 $R^{2}$ 和 $M E$ 损失最大值也都出现在率定效果较好的平山子流域, 该 子流域在率定时的 $R^{2}$ 和 $M E$ 均为 0.87 . 进一步分析在率定效果最差的星丰子流域上的效率损失. 星丰子 流域, 率定时的 $R^{2}$ 和 $M E$ 均为 0.72 , 算术平均值对应的 $R^{2}$ 和 $M E$ 损失值分别为 0.06 和 0.15 , 流域面积权 重平均值对应的为 0.07 和 0.18 , 泰森插值平均值对应的为 0.06 和 0.17 , 三者相比，也没有明显的差异. 因 此, 全局平均法中, 面积权重平均值和泰森多边形插值后平均并不能明显改进算术平均值估计参数的模 拟效果.

最后，用代理流域法中无资料流域参数估计效果稍好的九州子流域的参数和全局平均法中的算术 平均值进行比较, 同样选择率定效果最差的星丰子流域来作为参考. 当把九州子流域的参数用于估计星 丰子流域的参数时, $R^{2}$ 和 $M E$ 损失值分别将达到 0.03 和 0.08 , 而用区域内子流域的参数的算数平均值来 估计, $R^{2}$ 和 $M E$ 损失值分别为 0.06 和 0.15 , 相差不大. 因此, 在东江流域中, 两种方法在估计无资料流域 的参数值时, 将会得到相似的模拟结果. 
表 5 三组流域平均值在 13 个子流域和整个东江流域中的应用

Tab.5 Application of three average values in 13 sub-basins and Dongjiang Basin

\begin{tabular}{|c|c|c|c|c|c|c|c|c|c|c|c|c|c|c|}
\hline \multirow{3}{*}{ 名称 } & \multicolumn{2}{|c|}{ 率定 } & \multicolumn{4}{|c|}{ 算术平均值 } & \multicolumn{4}{|c|}{ 面积权重平均值 } & \multicolumn{4}{|c|}{ 泰森插值平均值 } \\
\hline & \multirow{2}{*}{$R^{2}$} & \multirow{2}{*}{$M E$} & \multirow{2}{*}{$R^{2}$} & \multirow{2}{*}{$M E$} & \multicolumn{2}{|c|}{ EL } & \multirow{2}{*}{$R^{2}$} & \multirow{2}{*}{$M E$} & \multicolumn{2}{|c|}{ EL } & \multirow{2}{*}{$R^{2}$} & \multirow{2}{*}{$M E$} & \multicolumn{2}{|c|}{ EL } \\
\hline & & & & & $R^{2}$ & $M E$ & & & $R^{2}$ & $M E$ & & & $R^{2}$ & $M E$ \\
\hline 东坑 & 0.97 & 0.96 & 0.93 & 0.90 & 0.04 & 0.06 & 0.93 & 0.92 & 0.03 & 0.05 & 0.93 & 0.91 & 0.04 & 0.06 \\
\hline 红花塔 & 0.71 & 0.71 & 0.64 & 0.62 & 0.07 & 0.08 & 0.64 & 0.62 & 0.07 & 0.08 & 0.64 & 0.63 & 0.07 & 0.08 \\
\hline 九州 & 0.87 & 0.87 & 0.85 & 0.85 & 0.02 & 0.02 & 0.85 & 0.85 & 0.02 & 0.02 & 0.85 & 0.84 & 0.02 & 0.03 \\
\hline 蓝塘 & 0.85 & 0.85 & 0.83 & 0.83 & 0.02 & 0.02 & 0.83 & 0.83 & 0.02 & 0.03 & 0.84 & 0.83 & 0.02 & 0.02 \\
\hline 连平 & 0.86 & 0.86 & 0.84 & 0.83 & 0.01 & 0.03 & 0.84 & 0.83 & 0.01 & 0.03 & 0.84 & 0.83 & 0.01 & 0.02 \\
\hline 黎嶂峰 & 0.81 & 0.80 & 0.79 & 0.79 & 0.01 & 0.01 & 0.80 & 0.79 & 0.01 & 0.01 & 0.79 & 0.79 & 0.01 & 0.02 \\
\hline 平山 & 0.87 & 0.87 & 0.75 & 0.64 & 0.12 & 0.23 & 0.74 & 0.61 & 0.13 & 0.26 & 0.76 & 0.64 & 0.11 & 0.23 \\
\hline 胜前 & 0.71 & 0.71 & 0.68 & 0.66 & 0.03 & 0.05 & 0.68 & 0.66 & 0.03 & 0.05 & 0.68 & 0.66 & 0.03 & 0.05 \\
\hline 水背 & 0.79 & 0.78 & 0.77 & 0.75 & 0.03 & 0.03 & 0.77 & 0.75 & 0.02 & 0.03 & 0.76 & 0.74 & 0.03 & 0.04 \\
\hline 顺天 & 0.89 & 0.88 & 0.82 & 0.74 & 0.07 & 0.14 & 0.83 & 0.76 & 0.06 & 0.13 & 0.82 & 0.75 & 0.07 & 0.13 \\
\hline 桃西 & 0.78 & 0.77 & 0.75 & 0.74 & 0.04 & 0.03 & 0.75 & 0.73 & 0.03 & 0.04 & 0.75 & 0.73 & 0.03 & 0.04 \\
\hline 星丰 & 0.72 & 0.72 & 0.67 & 0.57 & 0.06 & 0.15 & 0.66 & 0.55 & 0.07 & 0.18 & 0.66 & 0.55 & 0.06 & 0.17 \\
\hline 岳城 & 0.81 & 0.81 & 0.80 & 0.74 & 0.01 & 0.07 & 0.80 & 0.72 & 0.02 & 0.09 & 0.80 & 0.73 & 0.01 & 0.08 \\
\hline 东江 & 0.86 & 0.86 & 0.82 & 0.58 & 0.04 & 0.27 & 0.81 & 0.54 & 0.05 & 0.31 & 0.81 & 0.55 & 0.04 & 0.31 \\
\hline
\end{tabular}

\section{4 结语}

将 $\mathrm{HBV}$ 模型应用于东江流域及其子流域中, 并且采用了代理流域法和全局平均法两种方法来进行 参数区域化, 用于估计东江流域无资料流域的模型参数, 得出的主要结论如下: 1)HBV 模型去掉融雪模 块适用于中国南部无降雪地区的日径流模拟，模拟的流量过程与观测值较为吻合; 2)交叉检验中, 较小的 $R^{2}$ 和 $M E$ 值对应的参数, 其转移效果不一定比较大的 $R^{2}$ 和 $M E$ 值对应的参数转移效果差; 3)全局平均法中, 面积权重平均值和泰森多边形插值后平均并不能明显改进子流域算术平均值估计无资料流域的模型参数 的模拟结果; 4)在东江流域中, 代理流域法和全局平均法都能有效用于无资料流域的模型参数值的估计, 两者效果相差不大.

研究得出了东江流域中无资料流域参数估计方法的一些有用的结论, 但也存在着不足. 例如, 如前 面所述, 代理流域法中, 参数转移效果不仅与流域自然背景差异有关, 而且还与模型参数的敏感性、不确 定性等有着极大的关系, 这些都有待于在以后的研究中进行.

致谢: 感谢香港中文大学陈永勤教授所提供的水文资料. 河海大学杨涛老师和中国科学院南京地理与湖 泊研究所的杨宏伟老师在资料的收集和整理方面给予大量的帮助，特在此感谢.

\section{5 参考文献}

[1] Arnell NW. A simple water balance model for the simulation of streamflow over a large geographic domain. J Hydrol, 1999, 217: 314-335.

[2] Xu CY. Testing the transferability of regression equations derived from small sub-catchments to a large area in central Sweden. Hydrol Earth Syst Sci, 2003, 7: 317-324.

[3] Sivapalan M, Takeuchi K, Franks SW et al. IAHS decade on predictions in ungauged basins (PUB), 2003-2012: Shaping an exciting future for the hydrological sciences. Hydro Sci J, 2003, 48(6): 867-880.

[4] Riggs HC. Low-flow investigations, 1972. Techniques of water resources investigation of the U.S. Geological Survey, Book 4. U.S. Geological Survey, Washington DC, Chapter B1, 1972. 
[5] Mosley MP. Delimitation of New Zealand hydrologic regions. J Hydrol, 1981, 49: 173-192.

[6] Klemes V. Operational testing of hydrological simulation. Hydrolog Sci J, 1986, 31: 13-24.

[7] Xu CY. Estimation of parameters of a conceptual water balance model for ungauged catchments. Water Resour Manage, 1999 , 13: $353-368$

[8] Guo S, Wang J, Yang J. A semi-distributed hydrological model and its application in a macroscale basin in China. In: Dolman AJ, Hall AJ, Kavvas ML et al eds. Soil-Vegetation-Atmosphere Transfer Schemes and Large-Scale Hydrological Models. IAHS Publ, 2001, 270: 167-174.

[9] Vandewiele GL, Elias A. Monthly water balance of ungauged catchments obtained by geographical regionalization. $J$ Hydrol, 1995, 170: 277-291.

[10] Burn DH, Boorman DB. Estimation of hydrological parameters at ungauged catchments. J Hydrol, 1993, 143: 429-454.

[11] Huang M, Liang X, Liang Y. A transferability study of model parameters for the variable infiltration capacity land surface scheme. Journal of Geophysical Research, 2003, 108(D22)(doi:10.1029/2003JD003676).

[12] Seibert J. Regionalization of parameters for a conceptual rainfall-runoff model. Agr Forest Meteorol, 1999, 98-99: 279-293.

[13] Fernandez W, Vogel RM, Sankarasubramanian A. Regional calibration of a watershed model. Hydrolog Sci J, 2000, 45(5): 689-707.

[14] Kuczera G. Improved parameter inference in catchment models: Evaluating parameter uncertainty. Water Resour Res, 1983, 19(5): 1151-1162.

[15] Abdulla FA, Lettenmaier DP. Development of regional parameter estimation equations for a macroscale hydrologic model. $J$ Hydrol, 1997, 197: 230-257.

[16] Hundecha Y, Bardossy A. Modeling of the effect of land use changes on the runoff generation of a river basin through parameter regionalization of a watershed model. J Hydrol, 2004, 292: 281-295

[17] Gotzinger J, Bardossy A. Comparison of four regionalisation methods for a distributed hydrological model. J Hydrol, 2007, 333: 374-384.

[18] Braun LN, Renner CB. Application of a conceptual runoff model in different physiogra- phic regions of Switzerland. Hydrolog Sci J, 1992, 37(3): 217-231.

[19] Johansson B. The relationship between catchment characteristics and the parameters of a conceptual runoff model: a study in the south of Sweden, FRIEND. In: Proceedings of the Brau- nschweig Conference, October 1993, vol. 221.

[20] Kokkonen TS, Jakeman AJ, Young PC et al. Predicting daily flows in ungauged catchments: model regionalization from catchment descriptors at the Coweeta Hydrologic Laboratory, North Carolina. Hydrological Processes, 2003, 17: 2219-2238.

[21] Merz R, Bloschl G. Regionalisation of catchment model parameters. J Hydrol, 2004, 287: 95-123.

[22] Parajka P, Merz R, Bloschl G. A comparison of regionalization methods for catchment model parameters. Hydrol Earth Syst Sci, 2005, 9: 157-171.

[23] Bergstrom S. Development and application of a conceptual runoff model for Scandinavian catchments. SMHI RHO 7, Norrkoping, 1976: 134.

[24] Seibert J. HBV light version 1.3, User's manual, Uppsala University, Dept of Earth Science, Hydrology, Uppsala, 1998.

[25] Bergstrom S. The HBV model. In: Singh V ed. Computer models of watershed Hydrology. Water Resources Pub, 1995: 443-476. 